\title{
Convective Forcing Fluctuations in a Cloud-Resolving Model: Relevance to the Stochastic Parameterization Problem
}

\author{
G. J. ShuTts \\ Met Office, Exeter, United Kingdom \\ T. N. PALMER \\ ECMWF, Reading, United Kingdom
}

(Manuscript received 27 October 2005, in final form 3 April 2006)

\begin{abstract}
Idealized cloud-resolving model (CRM) simulations spanning a large part of the tropical atmosphere are used to evaluate the extent to which deterministic convective parameterizations fail to capture the statistical fluctuations in deep-convective forcing, and to provide probability distribution functions that may be used in stochastic parameterization schemes for global weather and climate models. A coarse-graining methodology is employed to deduce an effective convective warming rate appropriate to the grid scale of a forecast model, and a convective parameterization scheme is used to bin these computed tendencies into different ranges of convective forcing strength. The dependence of the probability distribution functions for the coarse-grained temperature tendency on parameterized tendency is then examined.

An aquaplanet simulation using a climate model, configured with similar horizontal resolution to that of the coarse-grained CRM fields, was used to compare temperature tendency variation (less the effect of advection and radiation) with that deduced as an effective forcing function from the CRM. The coarsegrained temperature tendency of the CRM is found to have a substantially broader probability distribution function than the equivalent quantity in the climate model.

The CRM-based probability distribution functions of precipitation rate and convective warming are related to the statistical mechanics theory of Craig and Cohen and the "stochastic physics" scheme of Buizza et al. It is found that the standard deviation of the coarse-grained effective convective warming is an approximately linear function of its mean, thereby providing some support for the Buizza et al. scheme, used operationally by ECMWF.
\end{abstract}

\section{Introduction}

Deep convection plays a key role in both the dynamics and thermodynamics of the atmospheric global circulation. On the other hand, neither global climate models nor indeed global operational numerical weather prediction (NWP) models can represent deep convective systems explicitly; rather, these models incorporate the effects of deep convection through parameterized bulk relationships between ensemblemean convective heat, moisture, and momentum fluxes and the instantaneous state of the atmosphere in a collocated vertical column.

While it is well known that the parameterized fluxes

Corresponding author address: G. J. Shutts, Met Office, Fitzroy Road, Exeter, Devon, EX1 3PB, United Kingdom.

E-mail: glenn.shutts@metoffice.gov.uk do not match the instantaneous fluxes that prevail in reality (Xu et al. 1992), neither the statistical nature of errors in parameterized fluxes nor their impact on weather forecasts and climate simulations is well quantified. Despite this, attempts to represent convective forcings in terms of some underlying probability distributions, rather than ensemble-mean bulk formulas, have been suggested. For example, Buizza et al. (1999) proposed adding a form of multiplicative noise to the equations of motion, where the multiplicative factor was applied to the ensemble-mean parameterized tendency. Lin and Neelin $(2000,2002)$ also experimented with stochastic perturbations to aspects internal to convective parameterization algorithms.

The Buizza et al. (1999) method (see also Palmer 2001) involves multiplying parameterization tendencies by a random number drawn from a uniform probability distribution function with a range between 0.5 and

DOI: $10.1175 /$ JCLI3954.1 
1.5. Space and time correlation is introduced by using the same random number in latitude/longitude boxes larger than the underlying model grid and holding the number fixed for over a certain number of time steps. Each forecast member has a different set of random numbers and this helps to correct a universal deficiency in current EPS systems-lack of spread. Buizza et al. adjusted the size of the boxes and time interval over which the random numbers are held constant in order to tune this "stochastic physics" scheme to get the best skill. Optimal skill in the European Centre for Medium-Range Weather Forecasts (ECMWF) ensemble prediction system (as measured by the Brier skill score for instance) was obtained for $10^{\circ}$ latitude/longitude boxes and 6-h time intervals.

More generally, Palmer $(1997,2001)$ proposed that representation of unresolved scales should be made using computationally efficient nonlinear stochasticdynamic models, for example, based on cellular automata (CA). Specific examples using such an approach have been proposed, for example, based on the Ising model (Majda and Khouider 2002; Khouider et al. 2003) and a specific CA onto which stochastically backscattered convectively forced fields are projected (Shutts 2005). Within this general framework, the use of simplified cloud-resolved models, embedded within each model grid box, is also relevant (Grabowski 2001; Randall et al. 2003).

However, a fundamental question concerns the validation of these more generalized approaches to the representation of unresolved scales. Data from field experiments are rather limited in both space and time and are generally inadequate to derive quantitative models for the statistics of instantaneous convective forcing. An alternative approach, one followed in this paper, is to use cloud-resolving models (CRMs) as estimates of truth. For instance, Pauluis and Garner (2006) used a CRM at different horizontal resolutions to determine the robustness of certain convective statistics (e.g., probability distributions of vertical velocity and cloud ice mixing ratio) and found a surprising degree of insensitivity-even at fairly coarse resolutions ( 2 to $50 \mathrm{~km}$ grid lengths). A rescaling of the vertical velocity based on a simple parcel model was shown to explain the weak horizontal-resolution sensitivity. Here we perform coarse-grained budgets of potential temperature using a coarse-graining length scale representative of current climate or NWP model grids. An initial step in this direction was reported by Shutts and Palmer (2004).

The purpose of this paper is to quantify the fluctuations in coarse-grained convective forcing, based on integrations of a CRM configured to represent a portion of the Tropics. Estimates of the strength of these fluctuations as a function of the ensemble-mean parameterized tendency allows a first validation of the functional relationship proposed in Buizza et al. (1999). This CRM is also used to assess the validity of the corresponding functional relationship implied by the application of the statistical mechanics formalism to the convective forcing problem (Craig and Cohen 2006).

\section{Model configuration}

The simulations to be described have been carried out with version 2.3 of the Met Office Large Eddy Model (LEM). The model is nonhydrostatic and uses an Arakawa C-grid staggering in horizontal planes and Lorenz grid in the vertical with periodicity assumed in both horizontal directions. In all experiments, 50 vertical levels are deployed nonuniformly with height with resolution ranging from $150 \mathrm{~m}$ in the boundary layer to $500 \mathrm{~m}$ in the free troposphere and $800 \mathrm{~m}$ in the stratosphere. The Coriolis parameter is made a linear function of $y$ with the equator at $y=0$, thereby achieving an equatorial beta plane. The domain is $7680 \mathrm{~km}$ squared with horizontal grid lengths $\Delta x$ and $\Delta y$ equal to 2 and $10 \mathrm{~km}$, respectively: it effectively spans the latitude range $35^{\circ} \mathrm{N}$ to $35^{\circ} \mathrm{S}$ and about 70 degrees of longitude. The grid anisotropy is less extreme than that in Shutts and Palmer (2004) and probably not important to the coarse-grain statistics computed here since the coarse grid-box scale is at least an order of magnitude larger than $\Delta y$. No obvious undesirable effects were seen at the northern and southern boundaries associated with the jump in Coriolis parameter. The use of periodic boundary conditions for an equatorial beta plane is more fully described in Shutts (2006, hereafter SHU).

Two different advection schemes have been used in these simulations: the centered difference scheme of Piacsek andWilliams (1970) and the monotonicitypreserving "ULTIMATE" scheme of Leonard et al. (1993). The standard approach for the LEM is to use the Piacsek-Williams scheme in the prognostic equation for momentum and ULTIMATE for thermodynamic variables, that is, potential temperature perturbation $\theta^{\prime}$ and all $q$ variables (representing water mixing ratios in its various forms). Here it was found to be necessary to use the Piacsek-Williams scheme on all variables except $q$ variables, where ULTIMATE was used to preserve their positive-definite nature. An interesting, though apparently spurious, gravity wave instability developed above the simulated subtropical jet streams when the ULTIMATE scheme was used to advect $\theta^{\prime}$, and, although the model remained stable, the 
effect on the stratospheric temperature profile was unacceptable. This did not occur when the PiacsekWilliams scheme was used. The problem may relate to the decomposition of the potential temperature field into a height-dependent reference state and a perturbation, with the ULTIMATE advection scheme being applied to the perturbation alone.

The LEM's subgrid-scale turbulence model is based on the classical Smagorinsky-Lilly assumption that the stress tensor is proportional to the flow deformation rate tensor multiplied by the product of the square of a mixing length and the modulus of the deformation. The approach is extended by adding a moist Richardsonnumber-dependent factor with different functional forms for heat and momentum. The moist Richardson number is based on a definition used by MacVean and Mason (1990). The mixing length is height dependent, decreasing from a chosen value outside the boundary layer (here $500 \mathrm{~m}$ ) to $k z_{0}$ at the surface where $k(=0.4)$ is the von Kármán constant and $z_{0}$ is the roughness length, chosen here to be $5 \times 10^{-3} \mathrm{~m}$. In view of the grid anisotropy it was decided to make the Smagorinsky-Lilly diffusion coefficient anisotropic by multiplying the diffusion terms associated with $y$ derivatives by $(\Delta y / \Delta x)^{2}$. Surface fluxes are determined, consistent with Monin-Obukhov similarity theory, given a fixed sea surface temperature (SST) distribution given by

$$
\operatorname{SST}(y)=301.0-6 \sin ^{2}\left(\frac{\pi y}{L}\right)+2 \sin \left(\frac{\pi x}{L}\right) \cos ^{2}\left(\frac{\pi y}{L}\right),
$$

where the units are kelvin and $L$ is the domain side length with $-L / 2<x, y<L / 2$. This pattern contains a zonally symmetric component with a maximum of 301 $\mathrm{K}$ at the equator falling to $295 \mathrm{~K}$ at the northern and southern limits of the domain together with a sinusoidal perturbation in $x$ of amplitude $2 \mathrm{~K}$. This crudely mimics the SST variation of the tropical Pacific ocean with a "warm pool" region on the left side of the domain.

In addition to the model's Smagorinsky-Lilly diffusion scheme it was felt beneficial to include horizontal diffusion with a constant diffusivity of $100 \mathrm{~m}^{2} \mathrm{~s}^{-1}$ in the $x$ direction and $100(\Delta y / \Delta x)^{2}$ (i.e., $2500 \mathrm{~m}^{2} \mathrm{~s}^{-1}$ ) in $y$. With the horizontal resolution used here convective updrafts tend to be one or two grid lengths wide, and therefore are improperly resolved. Horizontal diffusion is necessary when using the Piacsek-Williams scheme to help counteract the tendency to produce numerical noise in regions of tight gradients. To reduce spurious wave reflection at the model top (located at a height of $30 \mathrm{~km}$ ) the model's damping layer feature (which uses Newtonian relaxation on fluctuations about the hori- zontal area mean) was activated. The damping coefficient varies exponentially with height above $z=26 \mathrm{~km}$ with an equivalent relaxation time scale of $1000 \mathrm{~s}$ at the model top.

The model is run with a comprehensive three-phase cloud microphysics scheme that includes representations of rain, snow, graupel, ice cloud, ice number concentration, as well as water droplet clouds and vapor (Swann 1998). The radiation scheme was switched off for the purposes of these experiments and replaced with a fixed cooling profile-essentially a tropospheric cooling rate of $1.5 \mathrm{~K} \mathrm{day}^{-1}$ up to a height of $14 \mathrm{~km}$ and decaying to zero by $z=17 \mathrm{~km}$. This acts as a crude representation of infrared cooling primarily.

The initial state of the simulation was obtained from the zonally averaged state of an earlier simulation that used a north-south strip domain with uniform horizontal resolution of $1 \mathrm{~km}$ and with zonally symmetric SST (see SHU for details). This avoided a time-consuming spinup phase during which the convection effectively projects the underlying meridional SST variation onto the troposphere, and thermal wind adjustment causes the formation of two "subtropical" jet streams.

A "trade wind forcing (TWF) function" has been introduced into the model that takes the form of a fixed sink of westerly momentum, peaking at a height of 11 $\mathrm{km}$ and at latitudes $17^{\circ} \mathrm{N} / \mathrm{S}$ (Fig. 1). Its role is to represent the mean effect of Rossby wave drag due to the influx of wave action from the extratropics. The form and magnitude of the TWF is loosely based upon meridional/height sections of zonally averaged poleward momentum flux of westerly momentum derived from the 40-yr ECMWF Re-Analysis (ERA-40: Kållberg et al. 2005). The westerly deceleration, peaking as it does at a height of about $11 \mathrm{~km}$, contributes to driving the upper branches of two Hadley circulations. Since the depth-integrated TWF dominates the depth-integrated horizontal zonal momentum flux convergence, it is mainly responsible for the near-surface easterlies. In simulations without the TWF, the initial surface easterly flow (generated in response to the adjustment to thermal wind balance) tends to weaken and convection develops fairly uniformly with respect to latitude. With the TWF there is a tendency for convection to be suppressed poleward of about 20 degrees and enhanced equatorward of $15^{\circ} \mathrm{N}$ and $15^{\circ} \mathrm{S}$ (see SHU).

The simulation was run for five model days with daily dumps of model fields including tendencies of $u, v, w, \theta$, and $q$ (specific humidity). Hourly dumps were made between hour 100 and 120 and these, together with dumps at days 2, 3, and 4, were used to compute the convection statistics. 


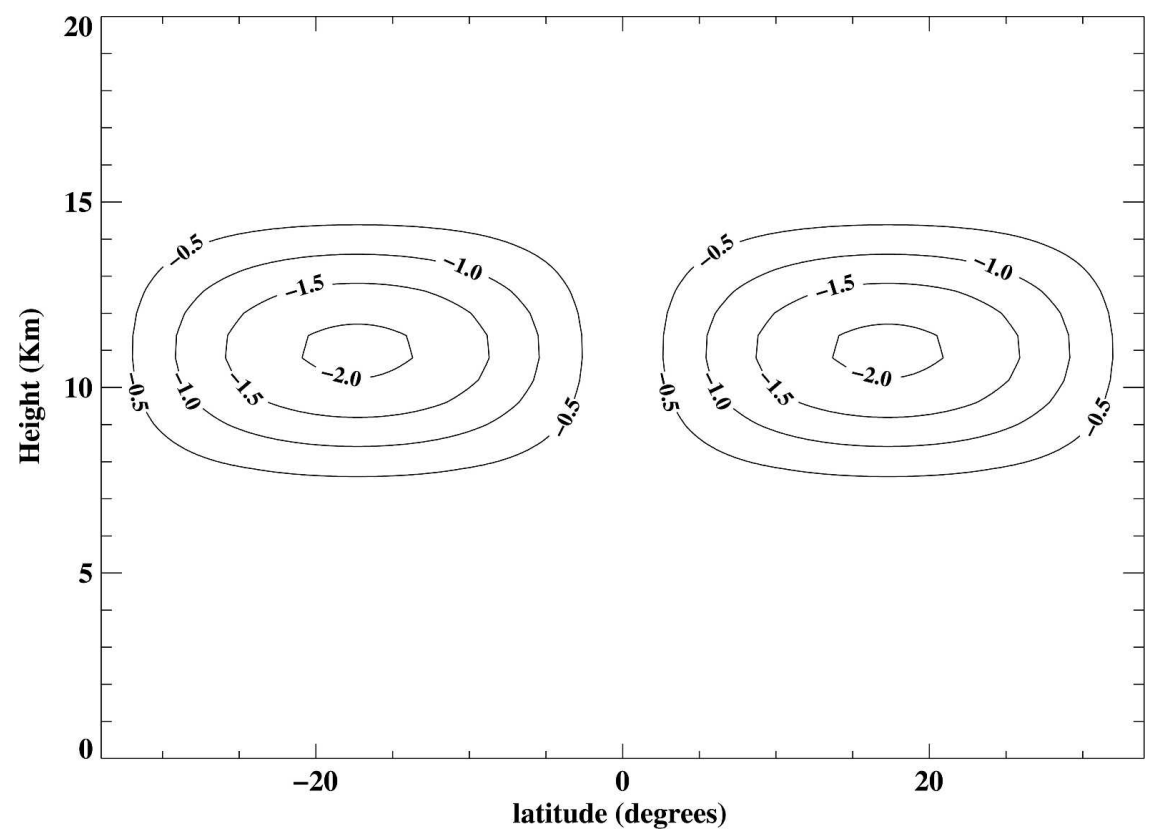

FIG. 1. Meridional-height cross section of the trade wind forcing function, which acts as a zonal momentum sink. Units: $\mathrm{m} \mathrm{s}^{-1}$ day $^{-1}$.

\section{Coarse-graining methodology}

The aim of this study is to use a sufficiently high resolution numerical model to simulate, with some realism, regions of tropical convection and to then coarsegrain the resulting fields and their associated tendencies to a grid resolution typical of current NWP or climate models. By the Reynolds-averaging approach outlined below it is possible to compute the nonadvective part of the total tendency that the corresponding coarse-grid model would see. Consider the thermodynamic equation written in terms of potential temperature:

$$
\frac{D \theta}{D t}=\frac{\partial \theta}{\partial t}+\mathbf{V} \cdot \nabla \theta=Q,
$$

where $Q$ represents the diabatic source term and rewrite this as

$$
\frac{\partial \theta}{\partial t}=-\mathbf{V} \cdot \nabla \theta+Q .
$$

Figure 2 illustrates a regular coarse grid used to perform the coarse-graining procedure with each coarse grid box containing nine fine grid boxes with integers $n$ and $j$ indexing the coarse and fine grid-box numbers, respectively. The value of a physical variable $\phi$ in the $j$ th fine grid box lying within the $n$th coarse grid box is denoted by $\phi_{n, j}$ and will represent a gridpoint value in the cloud-resolving model. The coarse-graining proce- dure involves averaging over all fine grid boxes within a coarse grid box leading to a coarse grid field $\bar{\phi}_{n}$ defined by

$$
\bar{\phi}_{n}=\frac{1}{J} \sum_{j=1}^{J} \phi_{n, j},
$$

where $J$ is the number of fine grid boxes within a coarse grid box. Note that, although the fine grid is portrayed as regular in Fig. 2, the CRM grid used here is anisotropic.

Now the discretized version of Eq. (3) for the cloudresolving model can be written as

$$
\frac{\partial \theta_{n, j}}{\partial t}=-\mathbf{V}_{n, j} \cdot \boldsymbol{\nabla} \theta_{n, j}+Q_{n, j} \cdot
$$

Averaging this equation over the fine grid boxes by summing over $j$ and dividing by $J$ gives

$$
\frac{\partial \bar{\theta}_{n}}{\partial t}=-\frac{1}{J} \sum_{j=1}^{J}\left(\mathbf{V}_{n, j} \cdot \boldsymbol{\nabla} \theta_{n, j}\right)+\bar{Q}_{n},
$$

and adding

$$
\overline{\mathbf{V}}_{n} \cdot \nabla \bar{\theta}_{n}
$$

to both sides gives an effective heating function $\left(\tilde{Q}_{n}\right)$ for the $n$th coarse grid box defined by

$$
\begin{aligned}
\frac{\partial \bar{\theta}_{n}}{\partial t}+\overline{\mathbf{V}}_{n} \cdot \boldsymbol{\nabla} \bar{\theta}_{n} & =\left[\overline{\mathbf{V}}_{n} \cdot \boldsymbol{\nabla} \bar{\theta}_{n}-\frac{1}{J} \sum_{j=1}^{J}\left(\mathbf{V}_{n, j} \cdot \boldsymbol{\nabla} \theta_{n, j}\right)\right]+\bar{Q}_{n} \\
& =\tilde{Q}_{n} .
\end{aligned}
$$




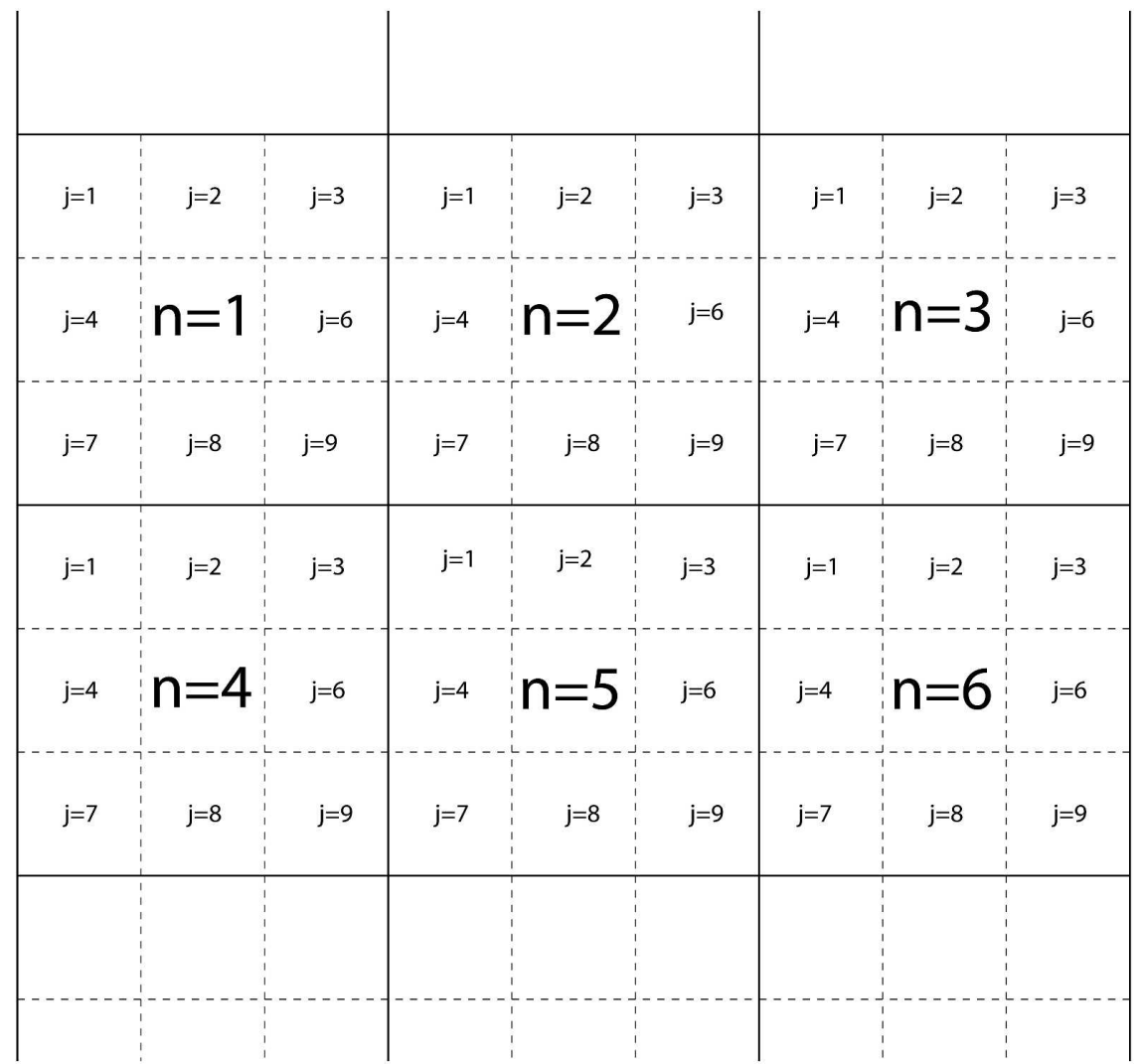

FIG. 2. Indexing of the coarse and fine grid boxes used to define the coarse-graining methodology. The coarse grid boxes are defined by the thick lines, with $n$ value specified in the center. The fine grid boxes, marked by dashed lines, have their $j$ values specified within and run from 1 to $J$ where $J=9$ in this illustration.

Equation (8) is the thermodynamic equation for the coarse-grained field $\bar{\theta}_{n}$.

Now consider the corresponding thermodynamic equation for an NWP or climate model whose grid exactly matches the coarse grid defined above, that is,

$$
\frac{\partial \theta_{n}}{\partial t}+\mathbf{V}_{n} \cdot \boldsymbol{\nabla} \theta_{n}+Q_{n}
$$

where the absence of the overbar distinguishes NWP model variables from their coarse-grained counterparts.

In practice $Q_{n}$ is composed of contributions from radiation $\left(Q_{R}\right)$, diffusion $\left(Q_{D}\right)$, and latent heat contributions associated with water phase changes $\left(Q_{L}\right)$. Of these contributions $Q_{L}$ is of most concern here. It can be split into an explicit term $Q_{\text {ls }}$ (where subscript "ls" signifies large scale and is diagnosed explicitly on the NWP model grid) and a contribution ( $Q 1)$ from subgrid-scale convective clouds determined from a parameterization scheme (using the commonly accepted definition of $Q 1$; e.g., Yanai et al. 1973).
For convenience the subscript $n$ will be dropped from $Q_{n}$ so that

$$
Q=Q_{R}+Q_{D}+Q_{\mathrm{ls}}+Q 1 .
$$

In most NWP models, a humidity-dependent partial cloud fraction concept permits condensational heating $Q_{\text {ls }}$ before the grid volume becomes saturated: $Q_{\text {ss }}$ may also contain a contribution that represents the evaporation of water droplets or ice that has been detrained from the convection parameterization scheme.

If $\mathbf{X}$ represents the state of the atmosphere at any instant, then $Q 1=Q 1(\mathbf{X})$ and it is also possible to define $\overline{Q 1}=Q 1(\overline{\mathbf{X}})$, where $\overline{\mathbf{X}}$ represents the coarsegrained state of all fields in the CRM. Using the CRM fields alone, we wish to examine the statistical relationship between the effective convective heating $\tilde{Q}_{n}$ and that given by the application of a convective parameterization scheme to the coarse-grained fields $(\overline{Q 1})$.

Ideally, one would want to group together all coarsegrained CRM grid columns that have similar vertical profiles (implying very similar profiles of $\overline{Q 1}$ ) and com- 
pute the probability distribution function of effective convective warming $\tilde{Q}_{n}$ for different classes of atmospheric conditions. Because of the very high dimensionality of this sample space the following compromise is made: $\overline{Q 1}$, evaluated at a particular height, will be used as a measure of the local strength of convective forcing in the CRM and probability density functions (PDFs) of $\tilde{Q}$ at the same height will be computed from subsamples of the coarse grid-box columns for different ranges of $\overline{Q 1}$. As will be seen later, the effective convective warming $\tilde{Q}$ is usually smaller than parameterized value $\overline{Q 1}$. This is because $\tilde{Q}$ better approximates $Q_{\mathrm{ls}}+Q 1$ in the context of an NWP model rather than $Q 1$ alone. In a tropical context $Q_{\mathrm{ss}}$ contains a large contribution from the evaporation and melting of condensate from stratiform cloud produced by the convective parameterization scheme. The sum of $Q_{\mathrm{ss}}$ and $Q 1$ largely balances the radiative energy sink in the Tropics above the boundary layer, but the relative sizes of the two terms is dependent on the parameterization scheme and its tuning. Here $\tilde{Q}$ contains the effect of both convective and "large-scale" diabatic heating/ cooling.

One possible way to determine a diabatic term equivalent to $Q_{1 \mathrm{~s}}$ in the CRM would be to pass the coarse-grained fields to the CRM's latent heating and cloud microphysics subroutines. However the resulting coarse-grid latent heating would not compare sensibly with $Q_{\text {ss }}$ from an NWP model because the CRM assumes grid-scale saturation before condensation occurs: NWP models allow for condensation when the volumemean humidity is less than $100 \%$ using a fractional cloud cover concept.

\section{Statistics derived from the tropical convection simulation}

To get some impression of the distribution of convection within the domain, the surface precipitation rate and near-surface temperature perturbation at day 4.5 are shown in Fig. 3. The logarithm (base 10) of precipitation rate is plotted with red denoting heavy rain (i.e., $\left.>40 \mathrm{~mm} \mathrm{~h}^{-1}\right)$, and very light rain $(\sim 0.1 \mathrm{~mm}$ $\mathrm{h}^{-1}$ ) is plotted blue. The heaviest convective rain occurs over the warmest SST in the western half of the domain and is organized into bands. Convection lines are located away from the "equator," which runs through the center of the domain. A double ITCZ feature appears early in the simulation in response to frictional convergence of the near-surface easterly winds but by day 4.5 it is a more broken feature and is located farther poleward over the warm pool region. The imprint of the SST pattern is clear in the near-surface temperature shown in the lower panel of Fig. 3, and its blotchy appearance is due to the effect of cold pools spreading out from active convection.

The PDF of rainfall intensity coarse-grained to a 120$\mathrm{km}$ grid for the whole domain is shown in Fig. 4. The $\log$ (probability) falls off linearly for rainfall intensities greater than $3 \mathrm{~mm} \mathrm{~h}^{-1}$, indicating an exponential tail. Figure 5 shows a histogram of the coarse-grained diabatic heating associated with latent heat exchange at $z$ $=9.4 \mathrm{~km}$ and extracted at $t=110 \mathrm{~h}$. At this scale $(120$ $\mathrm{km})$ the net latent heat source is frequently negative. Like real tropical clouds, convective anvils in the model extend over hundreds of kilometers away from the updrafts that generated them. Snapshots of the latent heating term in the middle to upper troposphere will therefore show substantial regions where ice crystals and snow evaporate into dry air. The asymmetry in the PDF implies a small net warming rate that balances the imposed cooling of $1.5 \mathrm{~K} \mathrm{~d}^{-1}$.

The corresponding PDF of the effective temperature forcing $\tilde{Q}$ at $z=9.4 \mathrm{~km}$ is shown in Fig. 6. At first sight the apparent symmetry of the curve about zero temperature tendency seems at odds with the primary effect of convective parameterization, which is to provide warming due to subsidence forced by the net upward convective mass flux. Although a large component of $\tilde{Q}$ comes from the direct effect of latent heating, cooling by evaporation also makes a big contribution, as is evident from Fig. 5.

One of the important issues of stochastic parameterization schemes used in ensemble prediction systems (e.g., Buizza et al. 1999) is the validity of "multiplicative noise," that is, the idea that the variance of parameterized tendencies should increase with the ensemblemean tendency-consistent with multiplying the deterministic parameterized tendencies by a random number.

To examine this question in greater depth it is instructive to form PDFs from subsamples of the coarsegrained data for which the degree of environmental convective stability varies. For instance, over the warm pool region of the domain one expects vigorous convection and strong fluctuations of convective tendency relative to the convection over cooler parts of the domain. As discussed in the previous section, PDFs of $\tilde{Q}$ will be conditioned on different ranges of $\overline{Q 1}$.

Coarse-grained fields of wind, temperature, and the mixing ratios of water vapor, liquid water, and ice from the model were supplied to the convection parameterization scheme of Bechtold et al. (2001). The scheme is a computationally efficient mass-flux-based parameterization whose conceptual framework was adapted from 

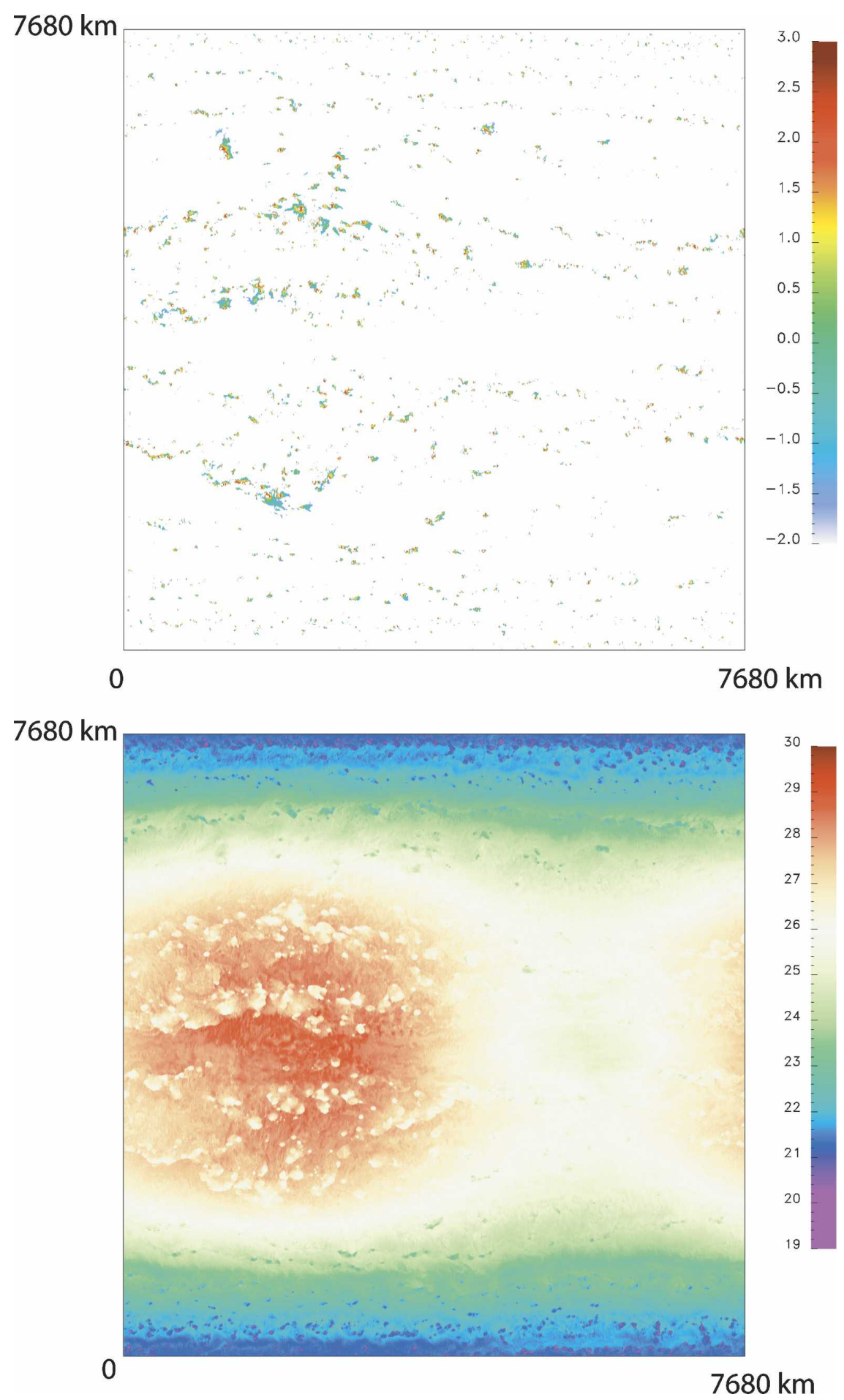

FIG. 3. (top) Log (precipitation rate in $\mathrm{mm} \mathrm{h}^{-1}$ ) plotted for day 4.5 of the simulation. (bottom) Temperature at $z=78 \mathrm{~m}$ at day $4.5(\mathrm{~K})$.

Kain and Fritsch (1990) but which includes a representation of cloud radii for deep and shallow clouds. The choice of this scheme was governed primarily by practical convenience rather than any particular features that might distinguish it from other convective parameterization schemes.

PDFs of $\tilde{Q}$ were formed from points whose corresponding parameterized convective temperature ten- 


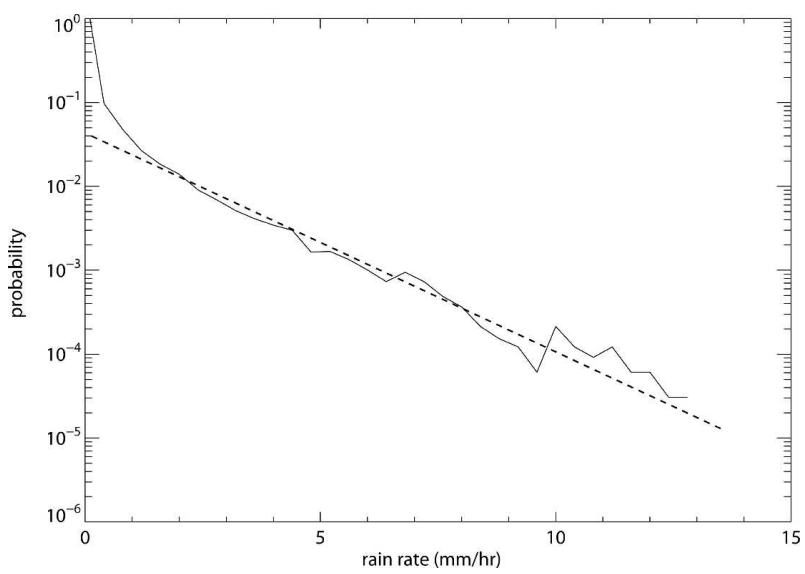

FIG. 4. Probability distribution function of surface precipitation rate for the whole domain, coarse-grained to $120-\mathrm{km}$ squares and computed from 20 instantaneous surface precipitation rate fields. The straight dashed line is provided just for reference.

dency $(\overline{Q 1})$ lies within specific $9 \mathrm{~K} \mathrm{day}^{-1}$ wide bin intervals. The results taken from 20 dumps at $z=9.4 \mathrm{~km}$ are shown in Figs. $7 \mathrm{a}-\mathrm{f}$ with related statistics summarized in Table 1. The bin used for Fig. 7a differs from the others in spanning the small range -0.1 to $+0.1 \mathrm{~K}$ day $^{-1}$ and essentially picks up points where the convective parameterization scheme has not been triggered. Notice the large number of points that go into this sample relative to the others, that is, about $94 \%$ of the coarse grid boxes (Table 1). The PDF is highly symmetrical for this subsample and that defined by the range $0.1<\overline{Q 1}<9 \mathrm{~K} \mathrm{day}^{-1}$ (Fig. $7 \mathrm{~b}$ ).

For the subsequent higher $9 \mathrm{~K}^{\text {day }}{ }^{-1}$ ranges of $\overline{Q 1}$ shown in Figs. 7c-f the asymmetry increases with a shift to more points on the positive side of the distribution.

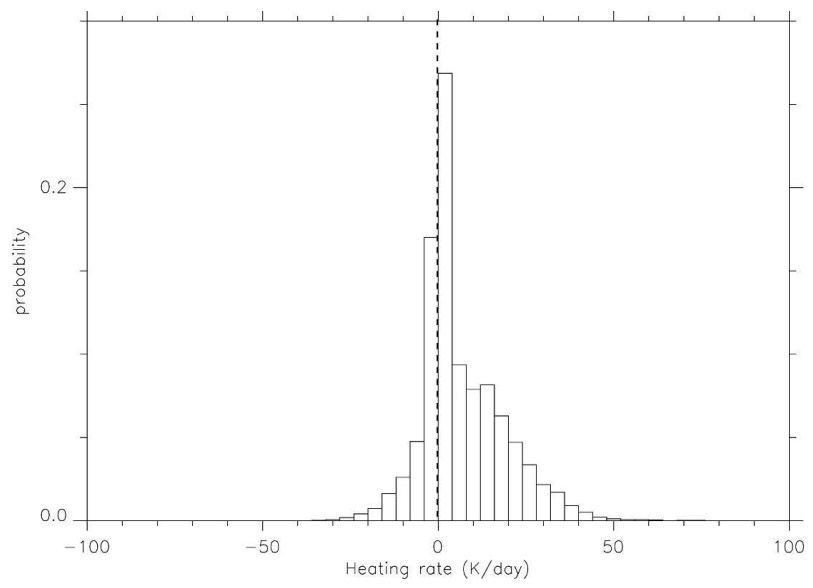

FIG. 5. Histogram of the coarse-grained latent heating/cooling (excluding the imposed cooling function) at a height of $9.4 \mathrm{~km}$ using $120-\mathrm{km}$ squares.

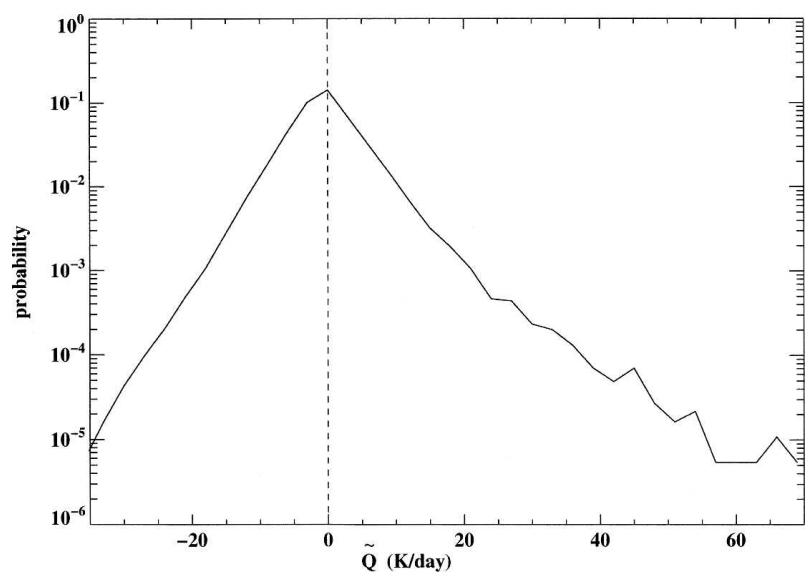

FIG. 6. PDF of effective temperature tendency $\tilde{Q}$ at $z=9.4 \mathrm{~km}$ on $120-\mathrm{km}$ squares obtained by the coarse-graining technique from 20 model dumps between day 2 and day 5 .

In the range $36<\overline{Q 1}<45 \mathrm{~K}$ day $^{-1}$ most of the points have positive temperature tendencies. Table 1 shows that, apart from the $36<\overline{Q 1}<45$ bin, the mean of $\tilde{Q}$ within each sample increases as the mean value of $\overline{Q 1}$ increases. The $36<\overline{Q 1}<45$ bin only contains 24 grid boxes, so the computed mean is unlikely to be accurate.

An interesting feature of the diagnostic application of a convective parameterization scheme to cloudresolving model fields is evident from the mean temperature tendency for coarse grid boxes with $-0.1<$ $\overline{Q 1}<0.1 \mathrm{~K} \mathrm{day}^{-1}$; it accounts for about $73 \%$ of the domain-averaged $\tilde{Q}$, implying that model convection is active in situations where the parameterization scheme is not triggered. The convection scheme was designed for use in an NWP model and not as a diagnostic tool. There are many parameters that are effectively "tunable" for optimal performance in the NWP context. For instance, the lowest level from which the parameterization calculation is started may be adjusted, and the resulting tendencies are found to be sensitive to this choice. Here this "start level" (located at $z=447 \mathrm{~m}$ ) was selected so that the domain horizontal average of $\overline{Q 1}$ at $z=9.4 \mathrm{~km}$ is closest to the corresponding average of $\tilde{Q}\left(\sim 1.17 \mathrm{~K} \mathrm{day}^{-1}\right)$. Note that it was not possible to retune that parameterization scheme to substantially reduce the number of convectively active coarse gridbox columns with zero parameterized convective tendency. This may also be interpreted as evidence for the failure of current convective parameterization methods based on grid-box mean fields.

Another way of presenting the relationship between $\tilde{Q}$ and $\overline{Q 1}$ is by plotting a scatter diagram (Fig. 8). The straight, dash-dot-dot line corresponds to $\tilde{Q}=\overline{Q 1}$, that is, where the convective parameterization scheme alone would have given the correct model temperature ten- 

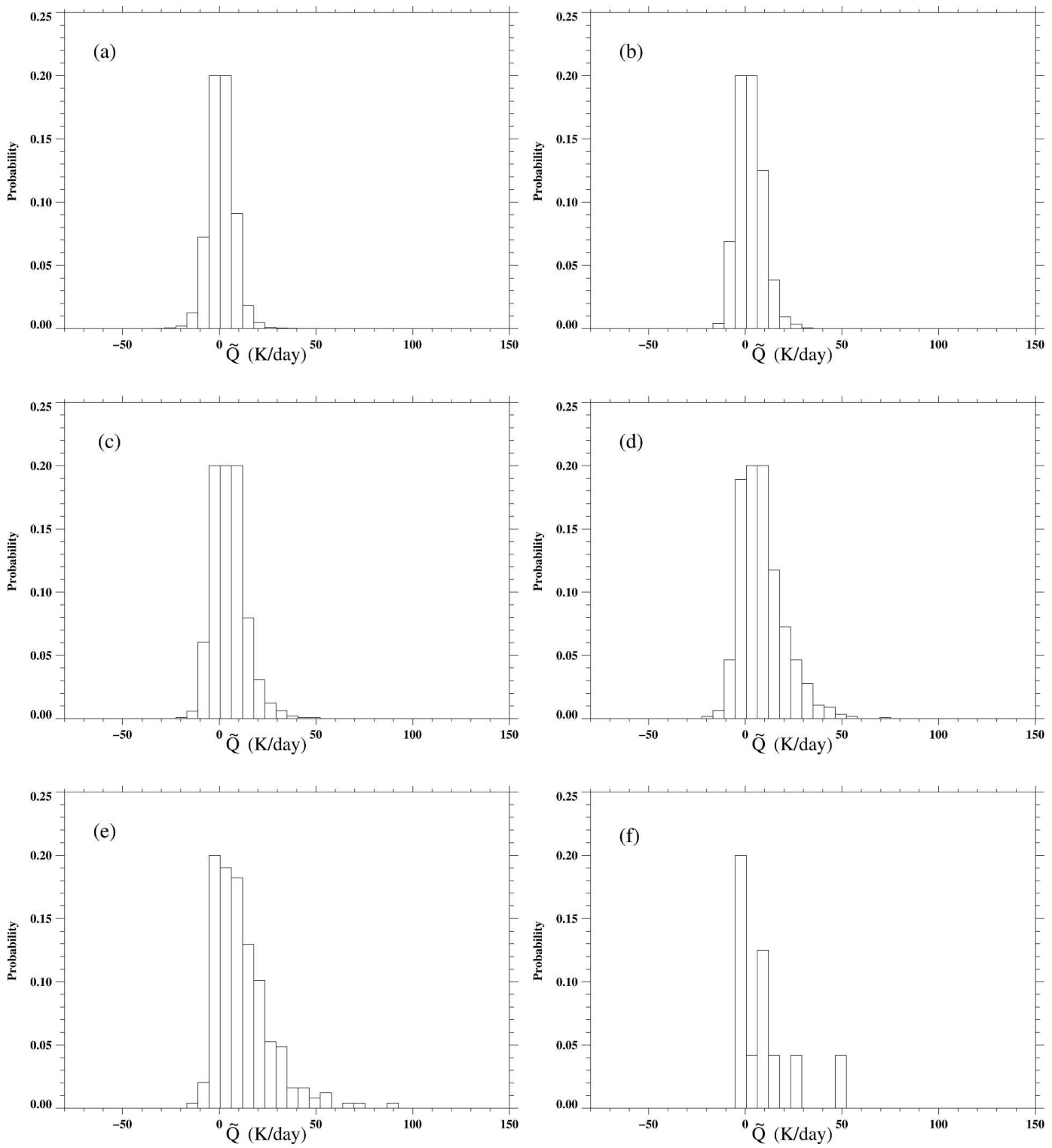

FIG. 7. Histograms of effective temperature tendencies obtained by averaging $\tilde{Q}$ at $z=9.4 \mathrm{~km}$ for a 120 -km grid. Each histogram is created from a subsample of all coarse boxes for which $\overline{Q 1}$ lies within the following ranges, i.e., (a) -0.1 to +0.1 ; (b) 0.1 to 9 ; (c) 9 to 18 ; (d) 18 to 27 ; (e) 27 to 36 ; and (f) 36 to $45 \mathrm{~K} \mathrm{day}^{-1}$.

dency in $120 \times 120 \mathrm{~km}$ grid boxes. The least squares fit through the cloud of points (denoted by $\tilde{Q}_{*}$ ) is indicated by the solid curve. As noted earlier, $\tilde{Q}$ is smaller than $\overline{Q 1}$ because $\overline{Q 1}$ does not account for explicit evaporation of cloud liquid water and ice. The highest density of points near, or somewhat above, the $\tilde{Q}=0$ axis is consistent with the peak of the PDF in Fig. 6. The broken vertical line at $\overline{Q 1}=0$ represents the $73 \%$ of points for which the convective parameterization scheme either does not trigger, or triggers but whose convective mass flux fails to reach a height of $9.4 \mathrm{~km}$. A least squares approach is also used to characterize the magnitude of fluctuations about the mean as represented by the dashed curve in Fig. 8. Fluctuations increase with $\overline{Q 1}$ but not as fast as $\tilde{Q}_{*}$ so the two curves cross at about $\overline{Q 1}=35 \mathrm{~K} \mathrm{day}^{-1}$. Neither the mean nor the fluctuation go to zero at $\overline{Q 1}=0$, consistent with the asymmetry and spread of the PDF of $\tilde{Q}$ about zero in Fig. 7a.

Given that the spatial autocorrelation scales of tropical convection are over $100 \mathrm{~km}$ (Ricciardulli and Sardeskmukh 2002), it is interesting to examine the PDFs of $\tilde{Q}$ on a coarser grid than $120 \mathrm{~km}$. Figure 9 shows conditioned PDFs for a coarse grid resolution of 
TABLE 1. Mean and standard deviation of the effective convective warming $(\tilde{Q})$ at $z=9.4 \mathrm{~km}$ for boxes belonging to different ranges of the parameterized convective warming $\overline{Q 1}$ at the same level. Mean $(\overline{Q 1})$ is the average of $\overline{Q 1}$ for grid boxes lying in the corresponding range; units in $\mathrm{K} \mathrm{day}^{-1}$. Coarse grid-box dimensions are $120 \mathrm{~km}$.

\begin{tabular}{ccccc}
\hline \hline$\overline{Q 1}$ range & $\begin{array}{c}\text { Mean } \\
(\tilde{Q})\end{array}$ & $\begin{array}{c}\text { Std dev } \\
(\tilde{Q})\end{array}$ & $\begin{array}{c}\text { Mean } \\
(\tilde{Q})\end{array}$ & $\begin{array}{c}\text { Number of } \\
\text { grid boxes }\end{array}$ \\
\hline-0.1 to 0.1 & 0.9 & 5.15 & 0.0 & 75078 \\
0.1 to 9.0 & 2.07 & 5.59 & 5.64 & 1745 \\
9.0 to 18.0 & 4.47 & 7.60 & 13.30 & 2409 \\
18.0 to 27.0 & 8.34 & 11.10 & 21.69 & 1116 \\
27.0 to 36.0 & 12.07 & 14.68 & 30.24 & 247 \\
36.0 to 45.0 & 4.87 & 11.36 & 38.74 & 24 \\
\hline
\end{tabular}

$320 \mathrm{~km}$. For the bin containing the points with $\overline{Q 1}=0$ (Fig. 9a) the mode of the distribution is now shifted well to the positive side of zero tendency and skewed toward positive values relative to the $120-\mathrm{km}$ coarse-graining results in Fig. 7. For the ranges $0<\overline{Q 1}<10,10<$ $\overline{Q 1}<20$ and $20<\overline{Q 1}<40 \mathrm{~K}$ day $^{-1}$ (Figs. 9b,c,d) the asymmetry is very marked, with only a small percentage of points having negative temperature tendency. This demonstrates that at the $320-\mathrm{km}$ box scale the contribution of cooling (presumably dominated by the evaporation of ice) is usually smaller than the latent heat release due to freezing since cloud systems are close to fitting within coarse grid boxes. The standard deviations of $\tilde{Q}$ in each range of $\overline{Q 1}$ are quite similar for all but the highest $\overline{Q 1}$ values, whereas the mean increases slowly and monotonically with mean $(\tilde{Q})$ (see Table 2). Interestingly, as was the case for $120-\mathrm{km}$ coarse boxes, the coarse-grain $\tilde{Q}$ values are much less than the associated $\overline{Q 1}$ values at this scale.

A scatterplot of $\tilde{Q}$ versus $\overline{Q 1}$ for the $320-\mathrm{km}$ grid is shown in Fig. 10. Coarse-graining at $320 \mathrm{~km}$ results in about 7 times less points than before, and this accounts for their sparsity. One thing that is immediately noticeable is the lack of clustering of points near $\tilde{Q}=0-$ consistent with the mode of the distributions in Fig. 9 moving to positive values. The reduced scatter of the points is consistent with the notion that deterministic convective parameterization becomes increasingly valid in the limit of large coarse grid-box size.

To relate the coarse-grain statistics of $\tilde{Q}$ to the behavior of a "live" convective parameterization scheme in an NWP model, the PDF of $Q_{D}+Q_{\mathrm{ls}}+Q 1$ from an aquaplanet configuration of the Met Office Unified Model (UM: Martin et al. 2006) was computed for different ranges of convective warming $\overline{Q 1}$. As discussed

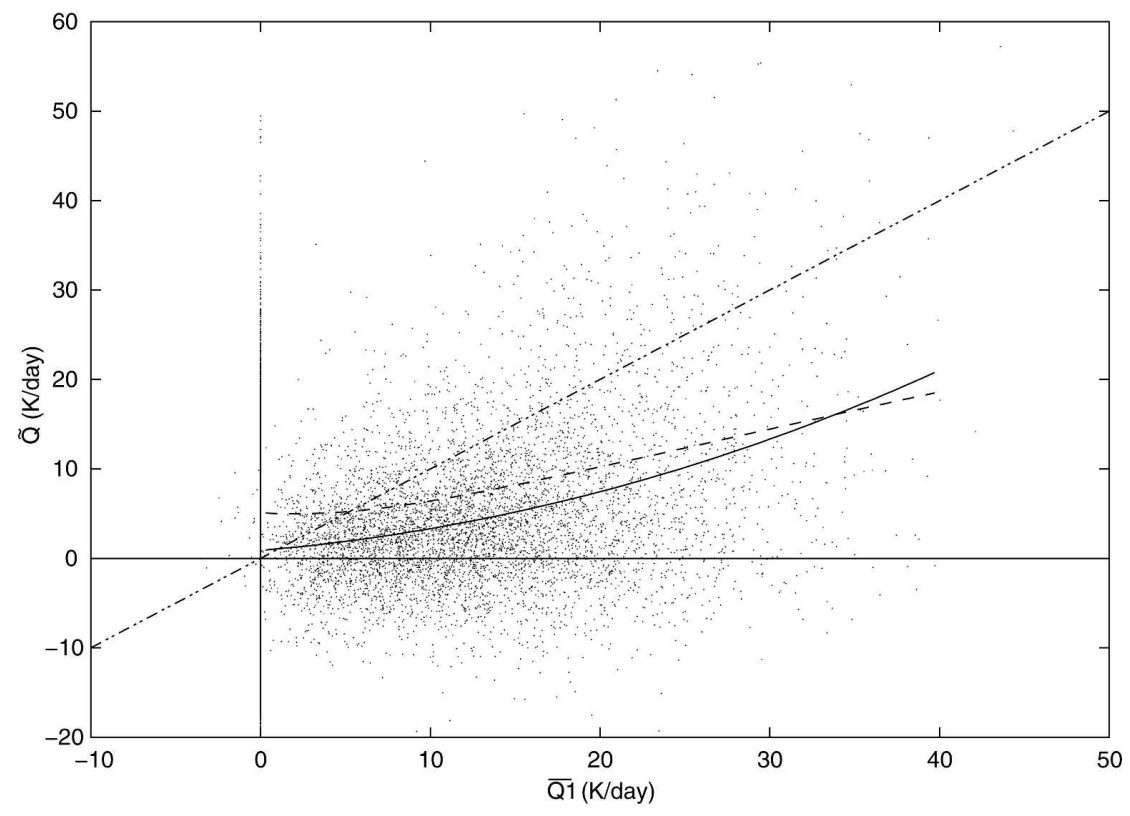

FIG. 8. Averaged coarse-grain effective temperature tendency $(\tilde{Q})$ vs parameterized temperature tendency $(\overline{Q 1})$ at $z=9.4 \mathrm{~km}$ taken from 20 instantaneous model dumps between day 2 and day 5 of the simulation. Most model dumps are at 1-h intervals although some gaps in the sequence occur. The solid line is a least squares fit to the points and the corresponding function will be denoted as $\tilde{Q}_{*}$. The dashed curve is a measure of the fluctuation about the mean (as represented by the solid curve) and is defined as follows. A least squares fit is obtained to the point variances $\left(\tilde{Q}-\tilde{Q}_{*}\right)^{2}$ and the square root of the resulting function is taken. 

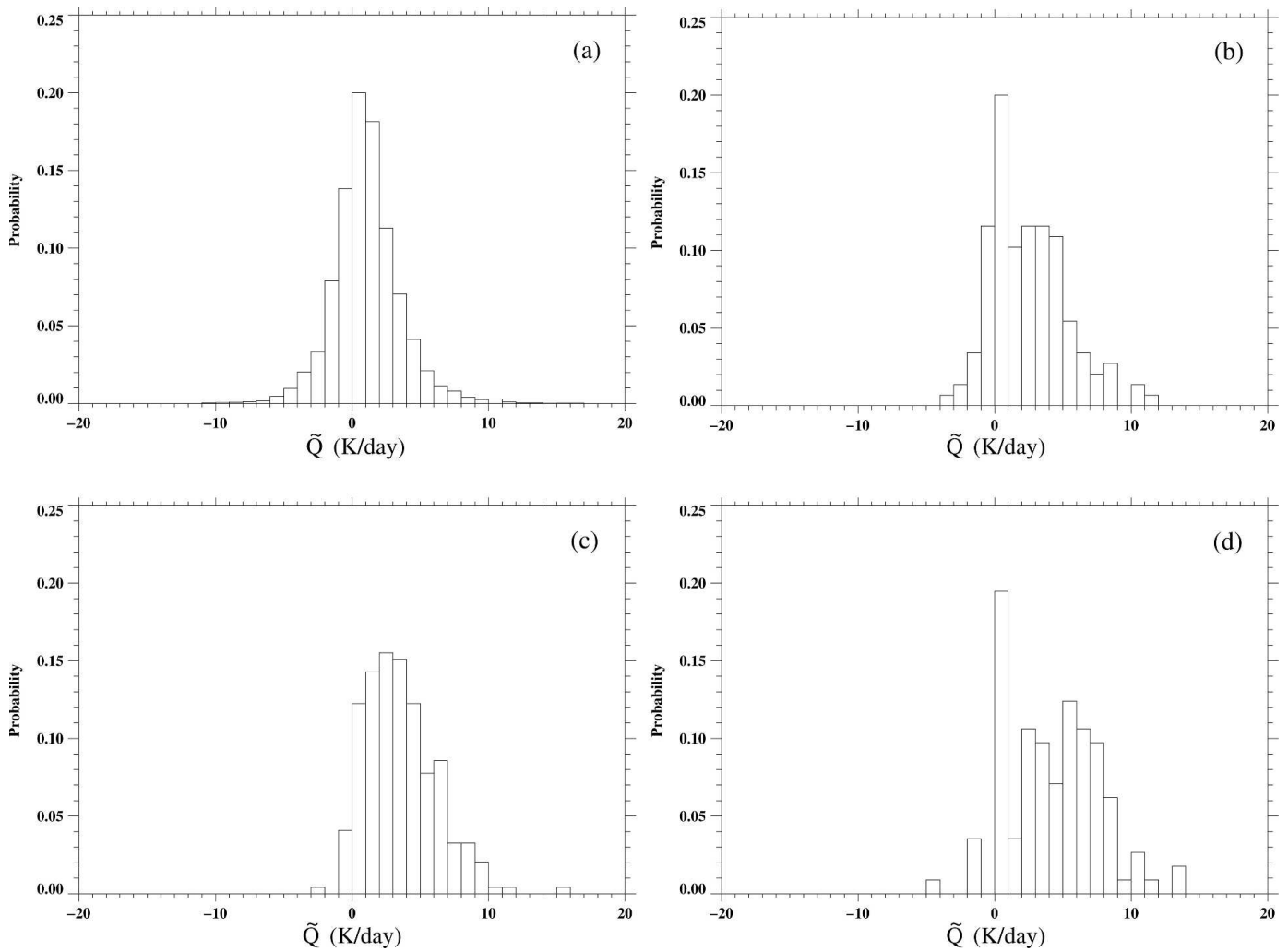

FIG. 9. As in Fig. 7 except for a coarse grid with 320-km square boxes. The corresponding ranges here are (a) -0.1 to +0.1 ; (b) 0.1 to 10.0 ; (c) 10 to 20 ; and (d) 20 to $40 \mathrm{~K} \mathrm{day}^{-1}$.

in section 3 , the effective convective warming rate $\tilde{Q}$ corresponds to $Q_{D}+Q_{\mathrm{ls}}+Q 1$ in a NWP model.

The SST distribution used was the control case of Neale and Hoskins (2001), which is functionally similar to that used in the CRM though with faster temperature falloff with latitude. Here the UM temperature tendencies between latitudes $\pm 30^{\circ}$ were taken from a version of the model with a mean horizontal grid length in the Tropics of $\sim 125 \mathrm{~km}$ and the contributions of advection and radiation were removed, thereby leaving parameterized convection, resolved-cale cloud microphysics and horizontal diffusion contributions only. The PDFs of $Q_{D}+Q_{\mathrm{ls}}+Q 1$ corresponding to those in Fig.

TABLE 2. As in Table 1 except for a coarse grid of box size 320 $\mathrm{km}$.

\begin{tabular}{ccccc}
\hline \hline$\overline{Q 1}$ range & $\begin{array}{c}\text { Mean } \\
(\tilde{Q})\end{array}$ & $\begin{array}{c}\text { Std dev } \\
(\tilde{Q})\end{array}$ & $\begin{array}{c}\text { Mean } \\
(\tilde{Q})\end{array}$ & $\begin{array}{c}\text { Number of } \\
\text { grid boxes }\end{array}$ \\
\hline-0.1 to 0.1 & 1.04 & 2.40 & 0.0 & 11014 \\
0.1 to 10.0 & 2.35 & 2.83 & 6.40 & 147 \\
10.0 to 20.0 & 3.54 & 2.69 & 15.91 & 245 \\
20.0 to 40.0 & 4.23 & 3.52 & 23.93 & 113 \\
\hline
\end{tabular}

7 are shown in Fig. 11 and are noticeably narrower than those derived from the CRM by coarse-graining.

In the case of the UM, scatter in the tendencies arises because of the variable effects of processes like resolved-scale evaporation of condensate and diffusion. Since the convection scheme is assumed to be deterministic, no random statistical fluctuation in convective forcing strength is involved, leading to narrower PDFs. As found for the coarse-grained convective warming tendency $\tilde{Q}$, the equivalent $\mathrm{UM}$ term, $Q_{D}+Q_{\mathrm{ls}}+Q 1$, is less than the mean of the parameterized convective warming $Q 1$ within the appropriate bin range (see Table 3. This is due to the combined effect of resolved latent cooling by evaporation and diffusion implicit in the semi-Lagrangian advection scheme, which oppose the positive parameterized temperature tendency.

Although the UM and CRM simulations are only roughly comparable, and the UM convective parameterization is different from the Bechtold et al. (2001) scheme used to bin $\tilde{Q}$, it is likely that the qualitative conclusions relating to the PDFs of convective forcing are valid; that is, the convection parameterization scheme appears to underestimate the magnitude of the statistical fluctuations in convection strength. 


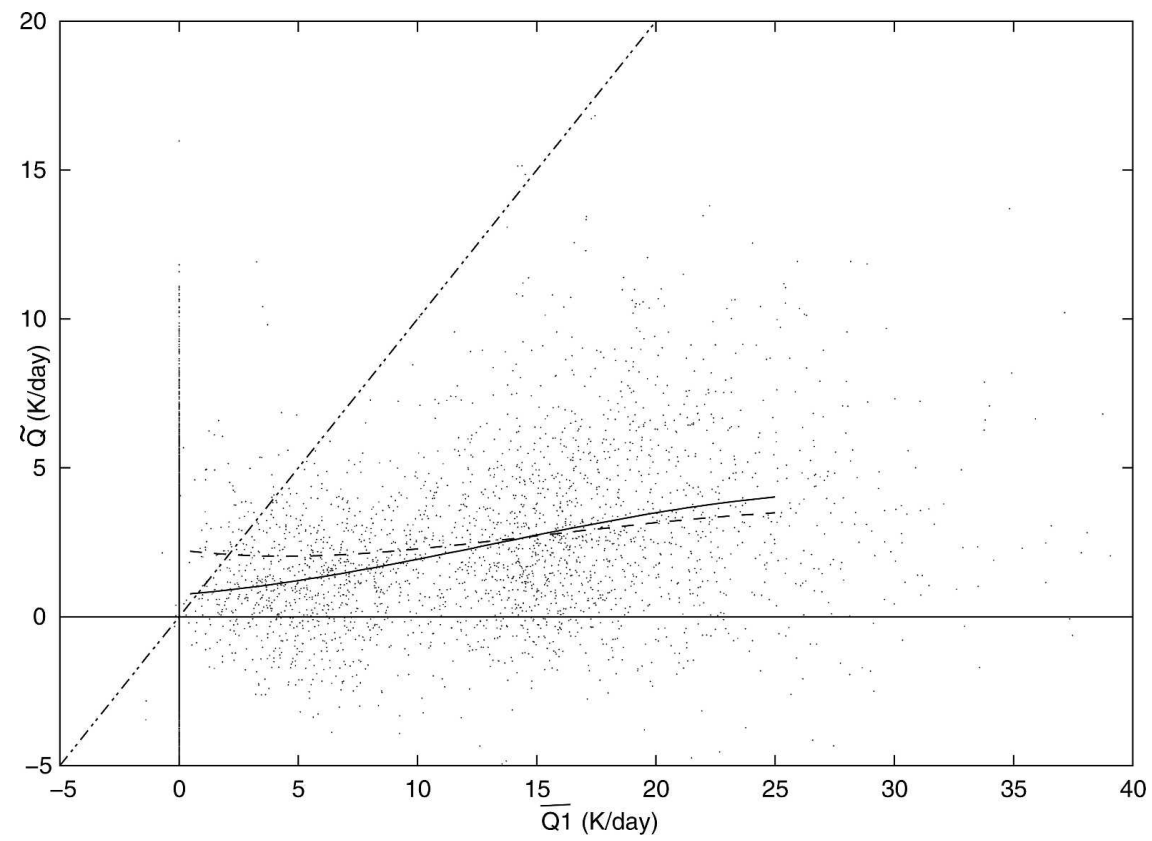

FIG. 10. As in Fig. 8 but with coarse graining to a $320-\mathrm{km}$ grid.

\section{Interpretation of the results with respect to a statistical theory and the ECMWF stochastic physics scheme}

The statistical mechanics view of convective cloud ensembles put forward by Craig and Cohen (2006) provides a useful reference point for interpreting some of the results given here. Their probability distribution function for the cloud mass flux can be expressed in the following form:

$$
\begin{aligned}
P\left(N_{*}\right)= & \left(\frac{\langle N\rangle}{N_{*}}\right)^{1 / 2} e^{-\left(\langle N\rangle+N_{*}\right)} I_{1}\left[2 \sqrt{N_{*}\langle N\rangle}\right] \\
& +\delta\left(N_{*}\right) e^{-\langle N\rangle}
\end{aligned}
$$

where $\langle N\rangle$ is the ensemble-mean number of clouds in a certain area, $N_{*}=M /\langle m\rangle, M$ is the convective mass flux, $\langle m\rangle$ is the mean mass flux per convective cloud, and $I_{1}[\cdots]$ is the first-order modified Bessel function. Application of statistical mechanics requires that the ensemble-mean convective mass flux (which is effectively determined by large-scale forcing, e.g., net radiative cooling), is carried by many noninteracting deep convective clouds. The mass flux carried by individual clouds is a random variable given by a Boltzmann-like probability distribution function $p(m)$ :

$$
p(m)=\frac{e^{-m /\langle m\rangle}}{\langle m\rangle},
$$

where $m$ is the mass flux carried by an individual cloud. The theory can be reinterpreted with the mass flux being replaced for instance by the net latent heat release whose ensemble average must essentially match the large-scale forcing, or equivalently the surface precipitation rate. In that case $N_{*}$ could be redefined so that $N_{*}=P_{r} /\left\langle p_{r}\right\rangle$, where $P_{r}$ is the total precipitation rate over some area and $p_{r}$ is the mean precipitation rate coming from a single cloud.

The delta function contribution in Eq. (11) gives the probability of the area under consideration containing no clouds, that is, $e^{-\langle N\rangle}$. Now for the $120-\mathrm{km}$ coarse grid used here this probability is very high. The use of precipitation rate as a random variable requires the definition of a minimum threshold below which a convective cloud is deemed not to exist. This is because the model produces unmeasurably fine rainfall rates over significant areas, most of which are numerical in origin. A reasonable probability for no convective clouds to exist within an $120 \times 120 \mathrm{~km}$ area is about $95 \%$ so that $e^{-\langle N\rangle}=0.95$ and $\langle N\rangle \approx 0.05$, that is, a low mean cloud density. In this low $\langle N\rangle$ limit the Bessel function may be approximated using:

$$
I_{1}(z)=\frac{z}{2}+O\left[z^{3}\right] .
$$

(e.g., Abramowitz and Stegun 1972; 9.6.10) so that Eq. (11) reduces to

$$
P\left(N_{*}\right)=\left(\langle N\rangle+O\left[\langle N\rangle^{2}\right]\right) e^{-\left(\langle N\rangle+N_{*}\right)}+\delta\left(N_{*}\right) e^{-\langle N\rangle}
$$

and the PDF for $N_{*}>0$ is exponential. 

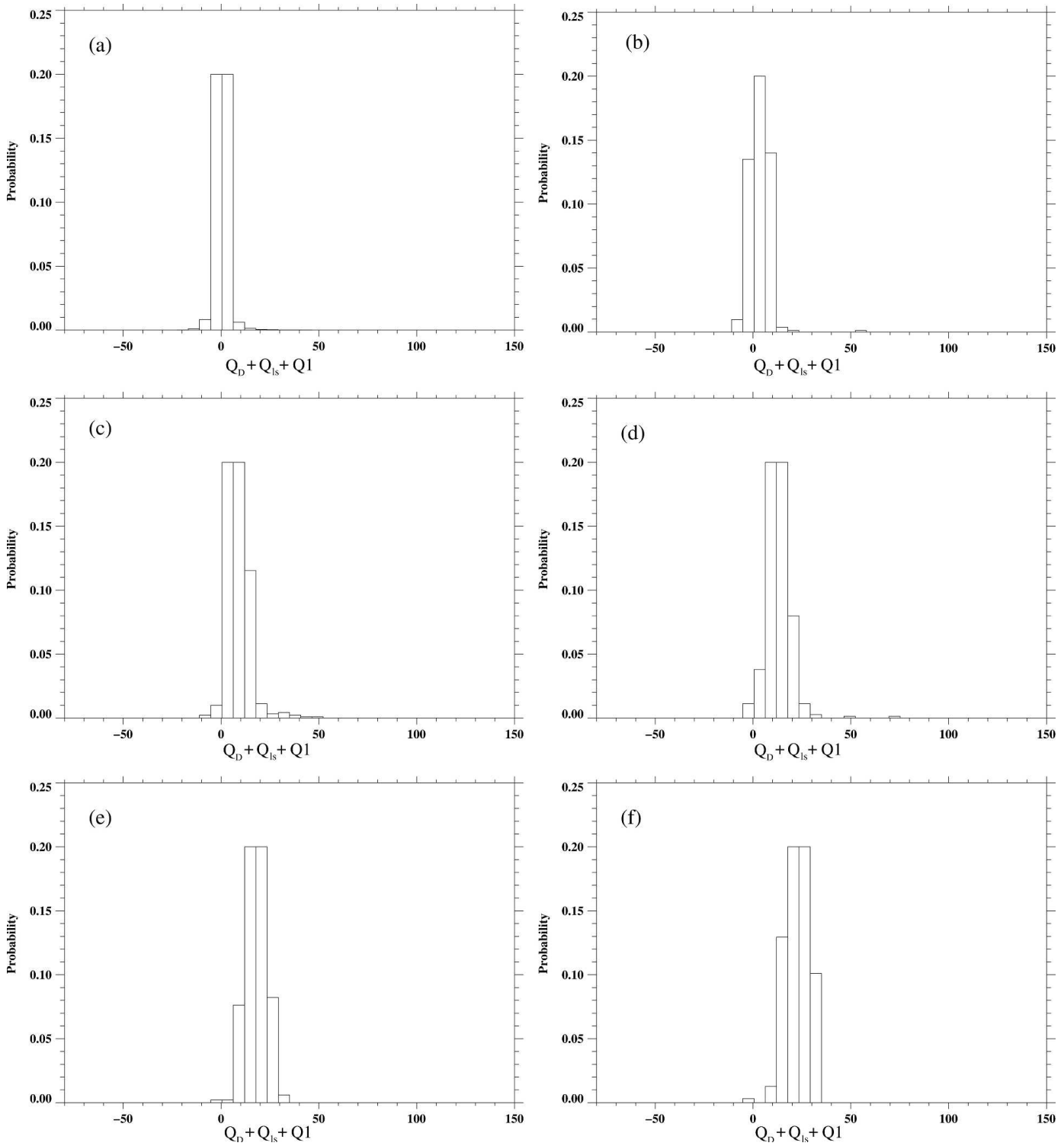

FIG. 11. As in Fig. 7 but from an aquaplanet simulation of the Met Office UM. The temperature tendency in this case is the full model tendency minus the contributions from radiation and advection, i.e., $Q_{\mathrm{D}}+Q_{\mathrm{ls}}+Q 1$ (data supplied by R. Stratton, Met Office).

The exponential tail seen in Fig. 3 is therefore consistent with the statistical theory of Craig and Cohen. For most of the time in the low-cloud density limit there are either no clouds, occasionally one cloud, and even more unlikely two clouds; so the probability distribution of mass flux tends to follow the exponential (Boltzmann) behavior of a single cloud. By integrating $\left(N_{*}-\right.$ $\langle N\rangle)^{2}$ weighted by the PDF over all $N_{*}$ the variance of $N_{*}$ can be shown to be $2\langle N\rangle$, implying that the mass flux variance itself is given by

$$
\left.<(M-\langle M\rangle)^{2}\right\rangle=2\langle m\rangle\langle M\rangle .
$$

TABle 3. As in Table 1 except from the Unified Model: $Q_{D}+$ $Q_{\mathrm{ls}}+Q 1$ is the UM equivalent of $\tilde{Q}$ in the coarse-graining calculation.

\begin{tabular}{ccccc}
\hline \hline$\overline{Q 1}$ range & $\begin{array}{c}\text { Mean } \\
\left(Q_{\mathrm{D}}+\right. \\
\left.Q_{\mathrm{ls}}+Q 1\right)\end{array}$ & $\begin{array}{c}\text { Std dev } \\
\left(Q_{\mathrm{D}}+\right. \\
\left.Q_{\mathrm{ls}}+Q 1\right)\end{array}$ & $\begin{array}{c}\text { Mean } \\
(Q 1)\end{array}$ & $\begin{array}{c}\text { Number of } \\
\text { grid boxes }\end{array}$ \\
\hline-0.1 to 0.1 & 0.08 & 2.25 & 0.0 & 79147 \\
0.1 to 9.0 & 3.22 & 3.55 & 4.87 & 822 \\
9.0 to 18.0 & 8.31 & 4.70 & 5.10 & 893 \\
18.0 to 27.0 & 12.53 & 5.10 & 22.17 & 713 \\
27.0 to 36.0 & 17.58 & 4.32 & 31.25 & 498 \\
36.0 to 45.0 & 23.12 & 5.10 & 40.29 & 317 \\
\hline
\end{tabular}


The point at which the standard deviation of the mass flux equals the ensemble mean mass flux is given by

$$
\sqrt{2} \sqrt{\langle m\rangle\langle M\rangle}=\langle M\rangle
$$

which simplifies to

$$
\langle N\rangle=\frac{\langle M\rangle}{\langle m\rangle}=2
$$

that is, when in the mean, two clouds occupy the given region.

If the convective heating rate $\tilde{Q}$ can be loosely associated with $\langle M\rangle$ and if $\overline{Q 1}$ represents a measure of convective forcing strength, then the crossover point of the solid and dashed lines in Figs. 8 and 10 [i.e., where std $\operatorname{dev} .(\tilde{Q})=$ mean $(\tilde{Q})]$ may be identified with the point where the magnitude of the mass flux fluctuation equals its ensemble mean. A literal interpretation of the theory implies that this happens when, on average, two deep convective clouds fit in a coarse box. As argued by Craig and Cohen, when the convective forcing strength increases, it is the number density of clouds that increases rather than the ensemble-average mass flux per cloud $\langle m\rangle$. Deterministic convective parameterization is therefore only likely to be valid for values of $\overline{Q 1}$ much larger than that at the crossover point in Figs. 8 and 10 where $\langle N\rangle=2$. Note that the value $\langle N\rangle=0.05$ deduced from consideration of precipitation rate intensities is a global average and $\langle N\rangle \geq 2$ only applies to a very small percentage of coarse boxes where the convective forcing is strong.

In the convective component of the ECMWF's stochastic physics scheme (Buizza et al. 1999) effectively assumes

$$
M=\alpha\langle M\rangle,
$$

where $\alpha$ is a random number with associated probability distribution function $P(\alpha)$ given by

$$
P(\alpha)= \begin{cases}1, & 0.5<\alpha<1.5 \\ 0, & \text { otherwise }\end{cases}
$$

The factor $\alpha$ is held fixed over $10^{\circ}$ latitude/longitude grid boxes, implying an equivalent coarse grid-box size of $\sim 1100 \mathrm{~km}$ [note that as of 1 February 2006 the operational ECMWF Ensemble Prediction System (EPS) runs at T399 resolution, equivalent to a horizontal grid length of about $50 \mathrm{~km}$ ].

The variance of the parameterized cloud mass flux is therefore given by

$$
\left\langle(M-\langle M\rangle)^{2}\right\rangle=\left\langle(\alpha-1)^{2}\right\rangle\langle M\rangle^{2},
$$

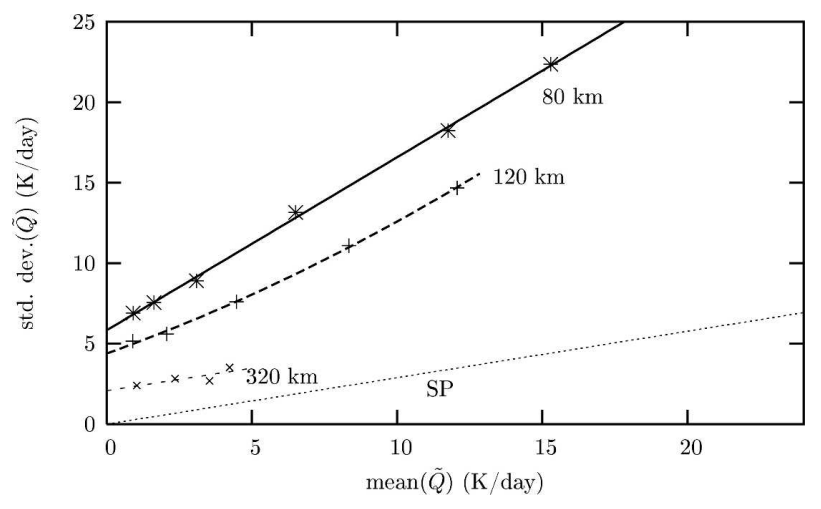

FIG. 12. Dependence of the standard deviation of $\tilde{Q}$ on its mean value within specified ranges of the associated parameterized convective warming ( $\overline{Q 1}$; as featured in Tables 1 and 2). Also shown are the results of coarse-graining to an $80-\mathrm{km}$ grid. Coarse-box sizes are given as labels next to the appropriate curves. The thin dotted line corresponds to the relationship between the mean and standard deviation of parameterized warming implied by the ECMWF stochastic physics scheme.

where

$$
\left\langle(\alpha-1)^{2}\right\rangle=\int_{0.5}^{1.5}(\alpha-1)^{2} d \alpha=\frac{1}{12},
$$

so

$$
\left\langle(M-\langle M\rangle)^{2}\right\rangle=\frac{1}{12}\langle M\rangle^{2} .
$$

Comparing the functional dependence of the variance of the mass flux against its mean in Eqs. (15) and (22), it can be seen that the Craig-Cohen theory suggests a linear dependence on $\langle M\rangle$, whereas the ECMWF stochastic physics scheme implies a square dependence. Figure 12 shows the mean and standard deviation of $\tilde{Q}$ for each bin at three coarse-graining resolutions. The data values for the $120-\mathrm{km}$ and $320-\mathrm{km}$ coarse grids are taken directly from Tables 1 and 2, and the curves are least squares fits of $\operatorname{std} \operatorname{dev}(\tilde{Q})$ to a quadratic polynomial in mean $(\tilde{Q})$.

At all three coarse-graining resolutions, the standard deviation of $\tilde{Q}$ is dominated by a linear dependence on mean $(\tilde{Q})$ although at the box size $120 \mathrm{~km}$ the dependence is somewhat faster than linear. As noted earlier, the nonvanishing standard deviation when mean $(\tilde{Q})=$ 0 relates to the existence of coarse boxes with net latent cooling as well as heating. For reference, the line representing the relationship between the standard deviation and the mean parameterized convective warming for the stochastically perturbed members of the ECMWF ensemble prediction system is also shown. In this case $\tilde{Q}$ should be interpreted as $Q 1$. 


\section{Discussion and conclusions}

It has been shown that coarse-grain cloud-resolving model PDFs are much broader than comparable PDFs from general circulation models. The coarse-graining technique has revealed that at the $120-\mathrm{km}$ scale (and smaller scales) the net latent heating is frequently negative (as judged from Fig. 5) and this translates into effective temperature tendencies that are also negative. Although this is only shown for $z=9.4 \mathrm{~km}$, it is true of most levels in the middle and upper troposphere of the model. At this scale the contributions of latent heat release (due to condensation/freezing) and evaporational cooling may not appear together in the same coarse box, so positive and negative values may dominate the box mean. Averaging over the lifetime of a convective system one expects a net latent heat warming consistent with the requirement that condensation should exceed evaporation in the presence of nonzero surface precipitation. Since convective parameterization schemes assume many cloud systems per grid box, the separate warming and cooling regions are hidden in the net positive convective warming tendencies. Therefore, the stochastic influence of convection parameterization should not merely be concerned with the variability in the size of convective mass fluxes and associated warming by subsidence but also the cooling associated with evaporating cloud condensate. In addition, latent cooling should be expected near and below the $0^{\circ} \mathrm{C}$ level where snow turns to rain.

The calculated coarse-grained effective temperature tendencies $(\tilde{Q})$ were sampled according to the strength of the associated parameterized convective tendency $(\overline{Q 1})$, and it was seen that their PDFs became more asymmetrical the higher the parameterized tendency and had greater variance. The standard deviation of $\tilde{Q}$ fluctuations was seen to have a linear dependence on mean $(\tilde{Q})$, although with an offset. In contrast the statistical mechanics theory of Craig and Cohen (2006) suggests that the standard deviation of $\tilde{Q}$ would vary as $\tilde{Q}^{1 / 2}$. This follows from the Poisson point process that governs the number of clouds for any fixed mass flux and the property that a Poisson random variable has a mean equal to its variance. Although the PDF for the convective mass flux is not a Poisson distribution [see Eq. (14)], it has similar properties. The linearity of the relationship between standard deviation and mean $\tilde{Q}$ lends support to simple multiplicative noise representations of stochasticity such as that of Buizza et al. (1999), although the offset should apparently be incorporated for better realism. The nonzero fluctuations in $\tilde{Q}$ when the mean is zero clearly poses a technical problem since it would be unreasonable to put convective tendencies into regions of the model atmosphere where deep convection would be impossible (e.g., over subzero continental regions in winter).

One drawback of the conditional sampling methodology used here is the inability of the parameterization scheme to identify all regions of convection when provided with the coarse-grained model fields. It is anticipated that this would be a general problem of the diagnostic application of convection parameterization schemes to flows that contain convective clouds and is not specific to the scheme used here. For instance, a radiosonde ascent made in close proximity to a deep convective system is unlikely to see instability, either because the boundary layer contains cool downdraft air or forced subsidence has warmed the midtroposphere and removed the CAPE.

Although a detailed study of the behavior of the UM convection scheme in the aquaplanet simulation is beyond the scope of this paper, these results suggest that the net parameterized temperature tendencies associated with convection have substantially smaller variance than those determined from the CRM. This is, of course, expected to be the case for a deterministic parameterization, and it is the effects of horizontal diffusion and resolved-scale latent heating that account for the spread of the UM effective temperature tendencies. On the other hand, the fluctuation in CRM temperature tendency is dominated by the statistical fluctuation in the number of convective cloud systems within the coarse grid box. It should be noted that from an implementation viewpoint the UM convective parameterization scheme is known to have excessive time-step-totime-step intermittency and, although this does not affect the instantaneous PDFs shown here, it could be regarded as a form of stochastic variability.

In addition to temperature tendency there are similar statistical issues associated with the parameterization of water vapor mixing ratio and vorticity tendencies. The coarse-graining methodology can also be used to deduce an eddy vorticity forcing field due to subgrid-scale Reynolds stresses associated with organized deep convection. Kinetic energy injected into the atmospheric mesoscale may migrate to larger scales as an inverse energy cascade of the kind described by Lilly (1983) and simulated by Vallis et al. (1997).

The results lend support to the use of the simple stochastic parameterization scheme of Buizza et al. (1999), used operationally in the ECMWF ensemble prediction system. However, this does not imply that more sophisticated nonlinear stochastic-dynamic models (Palmer 2001; Khouider et al. 2003; Shutts 2005; Randall et al. 2003) would not be more accurate still. 
Indeed the proposed methodology can be adapted to validate such schemes.

Future work is planned to study the PDFs of CRM convective temperature forcing projected onto the resolved scales of quasi-balanced motion, as these are more likely to be relevant to model flow impact. The goal of convective parameterization scheme development should be to ensure not only the correct gridpoint tendency PDFs but also an accurate representation of the spatial and temporal autocorrelation scales associated with mesoscale convective cloud organization.

Acknowledgments. We thank Tom Allen for considerable technical assistance during this work and also for many useful scientific discussions. Peter Bechtold supplied the convective parameterization scheme used for diagnostic analysis and we acknowledge much useful advice from him. We are also grateful to Rachel Stratton for providing the Unified Model temperature tendency statistics from an aqua-planet simulation for comparison with the CRM results. Roy Kershaw and Leon Hermanston have also provided useful suggestions during the course of this study.

\section{REFERENCES}

Abramowitz, M., and I. A. Stegun, 1972: Handbook of Mathematical Functions. Dover Publications, 1044 pp.

Bechtold, P., E. Bazile, F. Guichard, P. Mascart, and E. Richard, 2001: A mass flux convection scheme for regional and global models. Quart. J. Roy. Meteor. Soc., 127, 869-886.

Buizza, R., M. Miller, and T. N. Palmer, 1999: Stochastic representation of model uncertainty in the ECMWF ensemble prediction system. Quart. J. Roy. Meteor. Soc., 125, 2887-2908.

Craig, G. C., and B. G. Cohen, 2006: Fluctuations in an equilibrium convective ensemble. Part I: Theoretical formulation. $J$. Atmos. Sci., 63, 1996-2004.

Grabowski, W. W., 2001: Coupling cloud processes with the largescale dynamics using the cloud-resolving convection parameterization. J. Atmos. Sci., 58, 978-997.

Kain, J. S., and J. M. Fritsch, 1990: A one-dimensional entraining/ detraining plume model and its application in convective parameterizations. J. Atmos. Sci., 47, 2784-2802.

Kållberg, P., P. Berrisford, B. Hoskins, A. Simmons, S. Uppala, S. Lamy-Thépaut, and R. Hine, 2005: ERA-40 atlas. ERA-40 Project Report Series No. 19, ECMWF, Reading, United Kingdom, $191 \mathrm{pp}$.

Khouider, B., A. J. Majda, and M. A. Katsoulakis, 2003: Coarsegrained stochastic models for tropical convection and climate. Proc. Natl. Acad. Sci. USA, 100, 11 941-11946.

Leonard, B. P., MacVean, M. K., Lock, A. P., 1993: Positivitypreserving numerical schemes for multidimensional advection. NASA Tech. Memo. 106055 (ICOMP-93-05), 62 pp.

Lilly, D. K., 1983: Stratified turbulence and the mesoscale variability of the atmosphere. J. Atmos. Sci., 40, 749-761.

Lin, J.-B., and J. Neelin, 2000: Influence of a stochastic moist convective parametrization on tropical climate variability. Geophys. Res. Lett., 27, 3691-3694.
—, and — 2002: Considerations for stochastic convective parameterization. J. Atmos. Sci., 59, 959-975.

MacVean, M. K., and P. J. Mason, 1990: Cloud-top entrainment instability through small-scale mixing and its parameterization in numerical models. J. Atmos. Sci., 47, 1012-1030.

Majda, A. J., and B. Khouider, 2002: Stochastic and mesoscopic models for tropical convection. Proc. Natl. Acad. Sci. USA, 99, 1123-1128.

Martin, G. M., M. A. Ringer, V. D. Pope, A. Jones, C. Dearden, and T. J. Hinton, 2006: The physical properties of the atmosphere in the new Hadley Centre Global Environmental Model (HadGEM). Part I: Model description and global climatology. J. Climate, 19, 1274-1301.

Neale, R. B., and B. J. Hoskins, 2001: A standard test for AGCMs including their physical parametrizations: I: The proposal. Atmos. Sci. Lett., 1, 101-107.

Palmer, T. N., 1997: On parametrizing scales that are only somewhat smaller than the smallest resolved scales, with application to convection and orography. Proc. ECMWF Workshop on New Insights and Approaches to Convective Parametrization, Reading, United Kingdom, ECMWF, 328-337.

_ 2001: A nonlinear dynamical perspective on model error: A proposal for non-local stochastic-dynamic parametrization in weather and climate pres-diction models. Quart. J. Roy. Meteor. Soc., 127, 279-304.

Pauluis, O., and S. Garner, 2006: Sensitivity of radiativeconvective equilibrium simulations to horizontal resolution. J. Atmos. Sci., 63, 1910-1923.

Piacsek, S. A., and G. P. Williams, 1970: Conservation properties of convection difference schemes. J. Comput. Phys., 6, 392405.

Randall, D., M. Khairoutdinov, A. Arakawa, and W. Grabowski, 2003: Breaking the cloud parameterization deadlock. Bull. Amer. Meteor. Soc., 84, 1547-1564.

Ricciardulli, L., and P. D. Sardeshmukh, 2002: Local time and space scales of organized tropical convection. J. Climate, 15, 2775-2790.

Shutts, G. J., 2005: A kinetic energy backscatter algorithm for use in ensemble prediction systems. Quart. J. Roy. Meteor. Soc., 131, 3079-3102.

— , and T. N. Palmer, 2004: The use of high resolution numerical simulations of tropical circulation to calibrate stochastic physics scheme. Proc. ECMWF/CLIVAR Workshop on Simulation and Prediction of Intra-Seasonal Variability with Emphasis on the MJO, Reading, United Kingdom, ECMWF, 83-102.

_ - 2006: Upscale effects in simulations of tropical convection on an equatorial beta-plane. Dyn. Atmos. Oceans Sci., 42, $30-58$.

Swann, H., 1998: Sensitivity to the representation of precipitating ice in CRM simulations of deep convection. Atmos. Res., 48, 415-435.

Vallis, G. K., G. J. Shutts, and M. E. B. Gray, 1997: Balanced mesoscale motion and stratified turbulence forced by convection. Quart. J. Roy. Meteor. Soc., 123, 1621-1652.

Xu, K.-M., A. Arakawa, and S. Krueger, 1992: The macroscopic behavior of cumulus ensembles simulated by a cumulus ensemble model. J. Atmos. Sci., 49, 2402-2420.

Yanai, M., S. Esbensen, and J.-H. Chu, 1973: Determination of bulk properties of tropical cloud clusters from large-scale heat and moisture budgets. J. Atmos. Sci., 30, 611-627. 